\title{
Nonthermal Microwave Emission Features under the Plasma Ohmic Heating and Low-hybrid Current Drive in the FT- 2 Tokamak
}

\author{
V.V. Rozhdestvensky ${ }^{1, *}$, S.I. Lashkul ${ }^{1}$, V.V. Dyachenko ${ }^{1}$, E.M. Khilkevitch ${ }^{1}$, S.V. Krikunov ${ }^{1}$, L.A. Esipov ${ }^{1}$, \\ A.B. Altukhov ${ }^{1}$, D.V. Kouprienko ${ }^{1}$, A.Yu. Stepanov ${ }^{1}$, A.E. Shevelev ${ }^{1}$, S.V. Shatalin ${ }^{2}$ \\ ${ }^{1}$ Ioffe Institute, Russian Academy of Sciences, Russia \\ ${ }^{2}$ St.Petersburg State Polytekhnical University, Russia
}

Copyright $(\mathcal{C} 2015$ by authors, all rights reserved. Authors agree that this article remains permanently open access under the terms of the Creative Commons Attribution License 4.0 International License

\begin{abstract}
Study of behavior of accelerated electrons (AE) in the ohmically heated $(\mathrm{OH})$ plasma and low-hybrid current drive (LHCD) in tokamak has the practical interest especially for estimations of AE radiation energy losses and solving of the non-inductive CD problem for the ITER. Studies based on measurements of non-thermal microwave radiation (MR) intensity and hard X-ray (HXR) spectra are conducted in the FT-2 tokamak having large local magnetic ripples under LHCD in the omically heated $(\mathrm{OH})$ plasma with the fan instability excited. The paper presents experimental data first obtained in such conditions witnessing about some AE behavior features. Nature of MR abnormal intensity and short giant flashes together with the fast additional electron heating in the plasma core are discussed. The first high additional fast electron heating was registered together with the synchrotron radiation (SR) intensity increase. It was accompanied by short MR spikes observed in the narrow frequency range $(53 \div 78) \mathrm{GHz}$. They arise owing to the $\mathrm{AE}$ transverse energy and pitch angle increase under each crossing the cyclotron auto-resonance region and SR maser gain. It is proposed the heating mechanism owing to absorption of SR and Bernstein waves arising at linear transformation the extraordinary component of intensive MR spikes in the black plasma layers. It was found that the non-thermal MR of abnormal intensity arising during $\mathrm{OH}$ in the frequency range $(10 \div 40) \mathrm{GHz}$ is due to the fan instability development and the substantial local magnetic ripples. MR is accompanied by short giant flashes having a narrow frequency spectrum. Together with the SR growth the less intensive MR flashes appear in the range (57 $\div 75$ ) GHz. In our case it becomes possible the maser amplification of both SR and collective radiation. Appearance of the giant flashes may be initiated under transition of the maser - amplifier into the self - excitation regime, when low-frequency quasi- coherent MR flashes are generated.
\end{abstract}

Keywords Plasma, Accelerated Electrons, Synchrotron Emission, Cyclotron Autoresonance, Low-hybrid Current Drive

\section{Introduction}

On several tokamaks $[1 \div 6]$, short flashes of intense microwave radiation (MR) arising from the interaction of accelerated electrons with the harmonics of local toroidal magnetic ripples on the plasma periphery in the auto-resonance cyclotron mode has been discovered [7:9]. Under such conditions, maser amplification of synchrotron radiation (SR) proves to be possible in the frequency range corresponding to the width of the cyclotron auto-resonance. The flash intensity is determined by the density and energy of accelerated electrons, as well as the depth of magnetic ripples. The duration of the flashes depends on the confinement time of resonant conditions in the plasma. The first MR flashes of this nature have been found in the ohmic plasma heating on the ASDEX-U tokamak in the frequency range of $100 \div 120 \mathrm{GHz}$ at a relatively small depth of magnetic ripples, $\Delta B_{t} / B_{t} \sim 0.1 \%$, number of toroidal coils $N_{t}$ $=16$, and conditions unfavorable for the fan instability formation $[1,2]$. Similar MR flashes were recently registered on the FT-2 tokamak with a significantly greater depth of magnetic ripples, $\Delta B_{t} / B_{t} \sim 10 \%, N_{t}=24$, in experiments on generation of the lower hybrid current drive (LHCD) in the initially ohmic heated plasma $[4,10,11]$ with the excited fan instability.

\section{Experimental Evidence of the HXR and Synchrotron Radiation}


At high-frequency pumping of the plasma with low density $<n_{e}>\sim(1 \div 2) \times 10^{13} \mathrm{~cm}^{-3}$ electrons accelerated by the LH waves (LHW) and vortex electric field up to an energy of about $(4 \div 6) \mathrm{MeV}$ appear. For measurement of the superthermal and runaway electron energy the $\mathrm{NaI}(\mathrm{Tl})$ gamma-ray spectrometers with horizontal lines of sight are installed on tokamak FT-2 $\left(R=0.55 \mathrm{~m}, a=0.08 \mathrm{~m}, B_{T} \leq 3 \mathrm{~T}\right.$, $\left.I_{p l}=32 \mathrm{kA}, \quad<n>=1.910^{19} \mathrm{~m}^{-3}, f_{0}=920 \mathrm{MHz}\right)$ observing a poloidal diaphragm under various angles. Restored HXR spectra, recorded at $(20 \div 24) \mathrm{ms} \mathrm{OH},(30 \div 36) \mathrm{ms}$ LHCD and $(36 \div 42.4) \mathrm{ms} \mathrm{OH}$ (post RF-pulse) time slots, of the FT-2 shot are presented for central horizontal lines of sight in Fig. 1. The reconvoluted electron distribution functions (EDF) electron spectra using technique of [12] are represented for chosen time slots in the bottom figure.

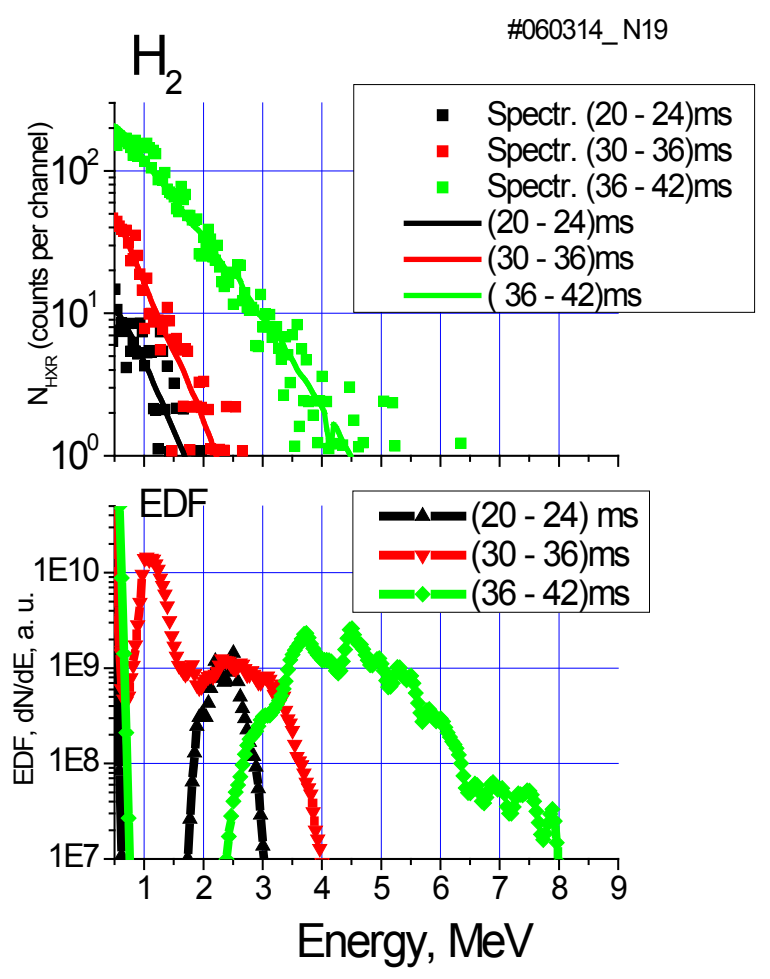

Figure 1. Measured (points) and restored (lines) HXR spectra (top figure). The reconvoluted electron distribution functions for three time slots of LHCD experiments (bottom figure).

The shape of the EDF reflects the evolution of the runaway generation process by LHW and vortex electrical field including primary and secondary generation modes. Such accelerated up to $4 \mathrm{MeV}$ electrons are able to enhance fan instability and effectively interact with the magnetic ripples in the plasma periphery. In this case, the SR intensity increases in the frequency range corresponding to the magnetic broadening of the first and second harmonics of the gyro-frequency of thermal electrons $(55 \div 78) \mathrm{GHz}$ and $(110$ $\div 156) \mathrm{GHz}$, correspondently, Fig. 2, 3, and short $(\sim 100 \mu \mathrm{s})$ flashes of microwave radiation presented in Fig. 4 appear caused by maser amplification of SR propagating in the same direction with runaway electrons.

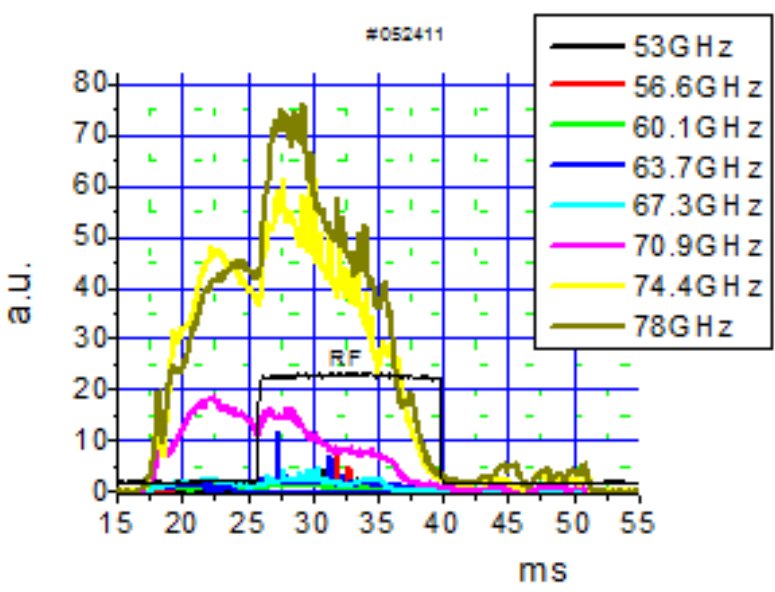

Figure 2. The intensity of SR increases in the frequency range corresponding to the magnetic broadening of the first harmonics

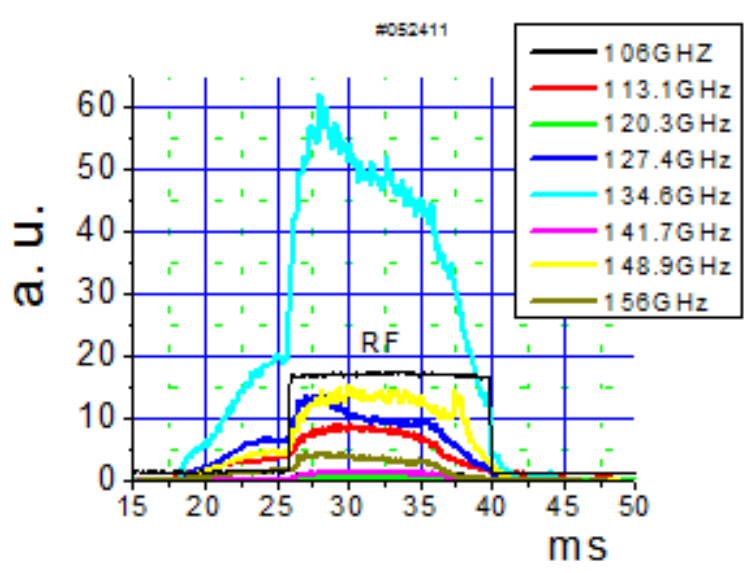

Figure 3. The intensity of SR increases in the frequency range corresponding to the magnetic broadening of the second harmonics

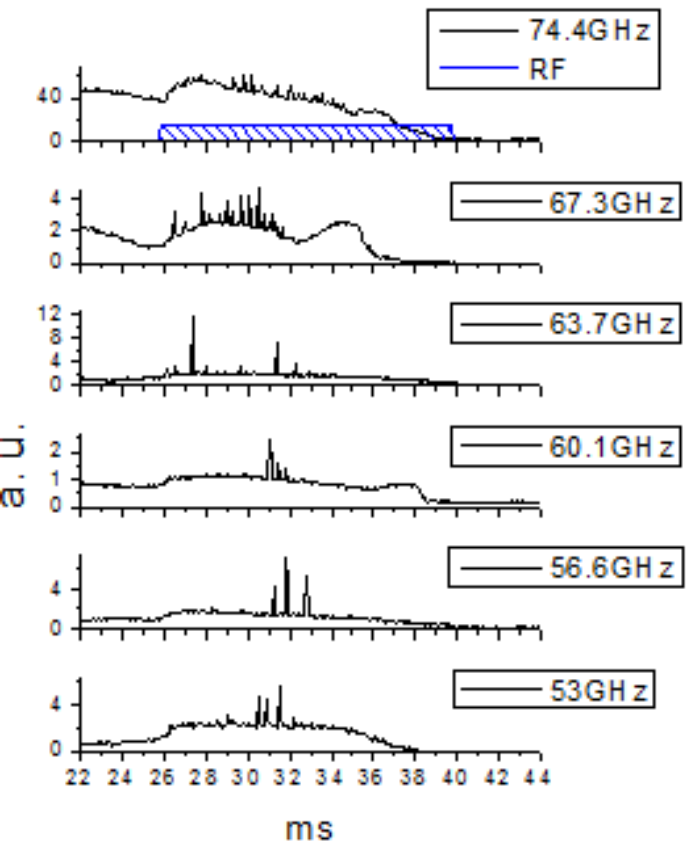

Figure 4. Short flashes of microwave radiation appear $(\sim 100 \mu \mathrm{s})$ presented in Fig. 2 caused by maser amplification of SR 
These spikes apparently result from the SR intensity growth under interaction of runaway electrons with significant toroidal magnetic field ripple harmonics in electron cyclotron resonance (ECR) conditions when the $\mathrm{AE}$ transverse energy and pitch angle increase at each crossing the resonance region. It turned out that the energy of runaway electrons accelerated by the residual vortex electric field cannot exceed a critical value determined by the auto-resonance condition, when the ECR frequency coincides with the $\mathrm{n}$-th harmonic of the toroidal magnetic field ripple one due to the SR intensity growth. As a result, runaway electrons will accumulate in the plasma periphery with a limited energy: $W_{\max }=e B_{t} R_{0} / n N_{t} m_{0} c$, where $\mathrm{e}$ is the electron charge, $\mathrm{m}_{0}$ is the electron mass, and $B_{t}, R_{0}$, and $N_{t}$ are the magnetic induction, major radius, and number of coils in the tokamak toroidal solenoid, respectively [13]. If these mono-energetic beam parameters are sufficient for microwave gain by the free electron flow under such resonance interaction, the cyclotron-auto-resonance maser (CARM) regime is realized.

$\# 012512$

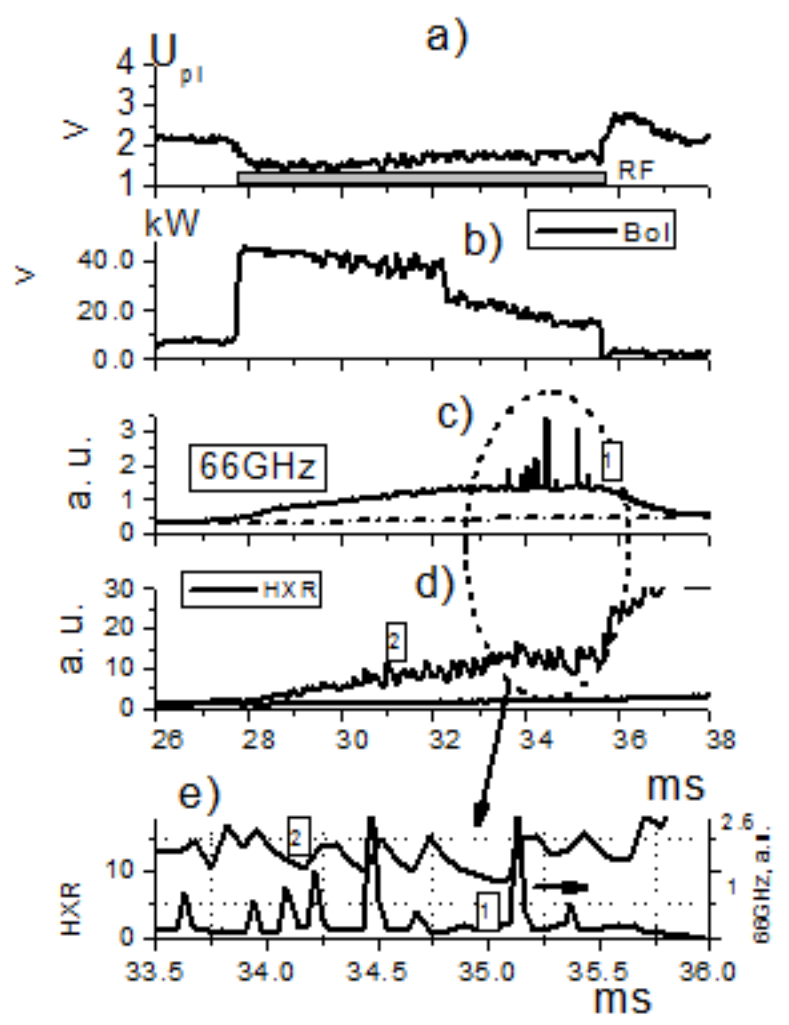

Figure 5. Variation of (a) voltage on the plasma column $\mathrm{U}_{\mathrm{Pl}}=2 \pi \mathrm{R} \mathrm{E}_{\theta}$, (b) bolometric signal, (c) synchrotron radiation at $66 \mathrm{GHz}$ (significant growth of this emission is accompanied by short spikes of microwave radiation related to maser spikes), and (d) hard X_ray (HXR) detector signal; (e) comparison of (1) microwave radiation spikes to (2) HXR detector signal waveform.

These emission pulses are manifested as a sequence of short MR spikes observed in a relatively narrow frequency interval $[1,2]$. To register MR in these experiments, we used
$2 \mathrm{~mm}$ and $4 \mathrm{~mm}$ heterodyne radiometers (HRs) with threshold sensitivity $P_{t h} \sim 10^{-12} \mathrm{~W}$, with an oversize transmission line and a circular horn antenna placed in the discharge chamber from the strong magnetic field side. The discharge chamber is a torus with sizes $R_{0}=0.55 \mathrm{~m}$ and $a=0.08 \mathrm{~m}$ having corrugated walls with corrugation step $d=8 \mathrm{~mm}$. During LHCD (Fig. 5a) significant growth of radiation losses has been observed, which is probably related to an increase in the intensity of synchrotron radiation from runaway electrons accelerated during the RF pimping pulse [4]. As can be seen from Fig. 5b (bolometric signal), the power of this radiation turns out to be close to the introduced RF power $\left(P_{\text {rad }} \sim\right.$ $\left.0.5 P_{R F}\right)$. This was accompanied by a significant increase in the intensity and energy of hard X_ray (HXR) photons (Fig. $5 \mathrm{~d})$ and the intensity of synchrotron radiation in the $\Delta f=$ (53-156) $\mathrm{GHz}$ frequency range. This growth was accompanied by short spikes of microwave radiation, Fig $5 \mathrm{c}$. As can be seen from Figs. 5c, $5 \mathrm{~d}$ and 5e, these spikes are well correlated with HXR ones that arise when locally trapped runaway electrons drift to emerge at the discharge chamber walls. In magnetic ripples of FT - 2 tokamak (with the number of turns $N_{t}=24$ in the toroidal solenoid), the value of $\delta B=\Delta B_{t} / B_{t}$ at the limiter reaches $10 \%$. Therefore, the accumulation of runaway electrons with the limited energy $3 \div 6 \mathrm{MeV}$ [4] in the LHCD regime under presence of a residual vortex electric field can ensure not only the additional generation of the high power SR in a broad frequency range but also the intensive MR flashing at the corresponding frequencies.

\subsection{Plasma Bulk Electrons Heating}

It turned out that for the plasma with $\left\langle n_{e}\right\rangle=1.2 \times 10^{13}$ $\mathrm{cm}^{-3}$ the occurrence of MR flashes during a RF-pulse is followed by a fast (within $2 \div 2.5 \mathrm{~ms}$ before end of the $14.5 \mathrm{~ms}$ RF pulse) additional heating of thermal electrons in the central plasma region $(r \leq 2 \mathrm{~cm})$ at temperature $T_{e}$ from 400 up to $550 \div 600 \mathrm{eV}$ and high-frequency pumping power $P_{r f} \sim 90 \mathrm{~kW}$ presented in Fig. 6 and Fig. 7 [4]. The average electron density of ohmic discharges in regime 1 and 2 were $<\mathrm{n}_{\mathrm{e}}>=1.2 \times 10^{13} \mathrm{~cm}^{-3}$ and $<\mathrm{n}_{\mathrm{e}}>=>1.4 \times 10^{13} \mathrm{~cm}^{-3}$, correspondently. It has been suggested that the additional electron heating in regime 1 could be caused either by the intense MR flashes and increased SR absorption in the plasma or improved core energy confinement.

For explanation by the first proposition we have analyzed the frequency dependence of the relative increment of the synchrotron radiation power in LHCD (see Fig. 2 and 3) using the formula:

$$
k=\frac{P(30 m s)-P(25 m s)}{P(25 m s)}
$$

where $\mathrm{P}(25 \mathrm{~ms})$ and $\mathrm{P}(30 \mathrm{~ms})$ are the signal levels at a given frequency prior to switch on ( $25 \mathrm{~ms})$ of the RF- heating pulse and in $5 \mathrm{~ms}$ later its switch on $(30 \mathrm{~ms})$, respectively. This analysis, Fig. 8, showed the presence of troughs in the 
vicinity of the following characteristic frequencies: $f_{\mathrm{Be}}=62 \mathrm{GHz}$ (the ECR, Bernstein wave) and upper hybrid resonance (UHR) $f_{U H R}=\sqrt{{f_{B e}{ }^{2}+{f_{P l}}^{2}}^{2}}=68 \mathrm{GHz}$, frequencies [14], where $\mathrm{fp}=28 \mathrm{GHz}-$ plasma frequency for $\mathrm{n}_{\mathrm{e}}=10^{13} \mathrm{~cm}^{-3}$. Thus during the LHCD generation, synchrotron microwave radiation can be absorbed by thermal electrons of plasma, which is evidenced by troughs at the characteristic frequencies.

Since high power microwave radiation is generated in the FT-2 tokamak, it can be suggested that the additional fast central heating of electrons can be related to the SR absorption in the black plasma layers and a linear transformation of the extraordinary component of electromagnetic microwave radiation to Bernstein waves in the UHR region and their absorption in the ECR region at $f \sim$ $(60-70) \mathrm{GHz}(\lambda=4 \mathrm{~mm})$ for $B_{\mathrm{t}}=1.95 \mathrm{~T}$ [14]. ASTRA code estimations show, that approximately $P_{R F}=10 \mathrm{~kW}$ need for electron central core heating from 400 up to $600 \mathrm{eV}$.

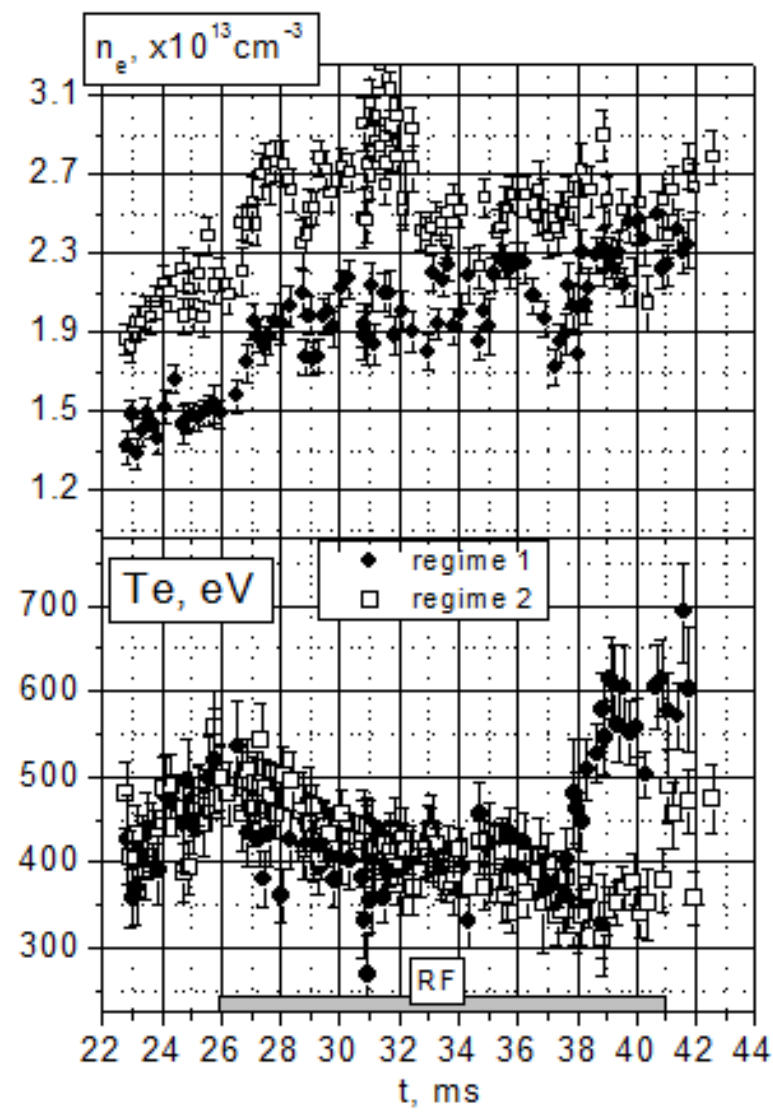

Figure 6. Variation of the (a) electron density $\mathrm{n}_{\mathrm{e}}(\mathrm{y}=0, \mathrm{t})$ and (b) electron temperature $T_{e}(y=0 \mathrm{~cm}, t)$ at the center of plasma column during LHCD generation in two regimes: (regime 1) at an average electron density of ohmic discharge $\left\langle\mathrm{n}_{\mathrm{e}}\right\rangle=1.2 \times 10^{13} \mathrm{~cm}^{-3}$; (regime 2) at average electron density of $\left\langle\mathrm{n}_{\mathrm{e}}\right\rangle=>1.4 \times 10^{13} \mathrm{~cm}^{-3}$. Data obtained by laser Thomson scattering diagnostics.

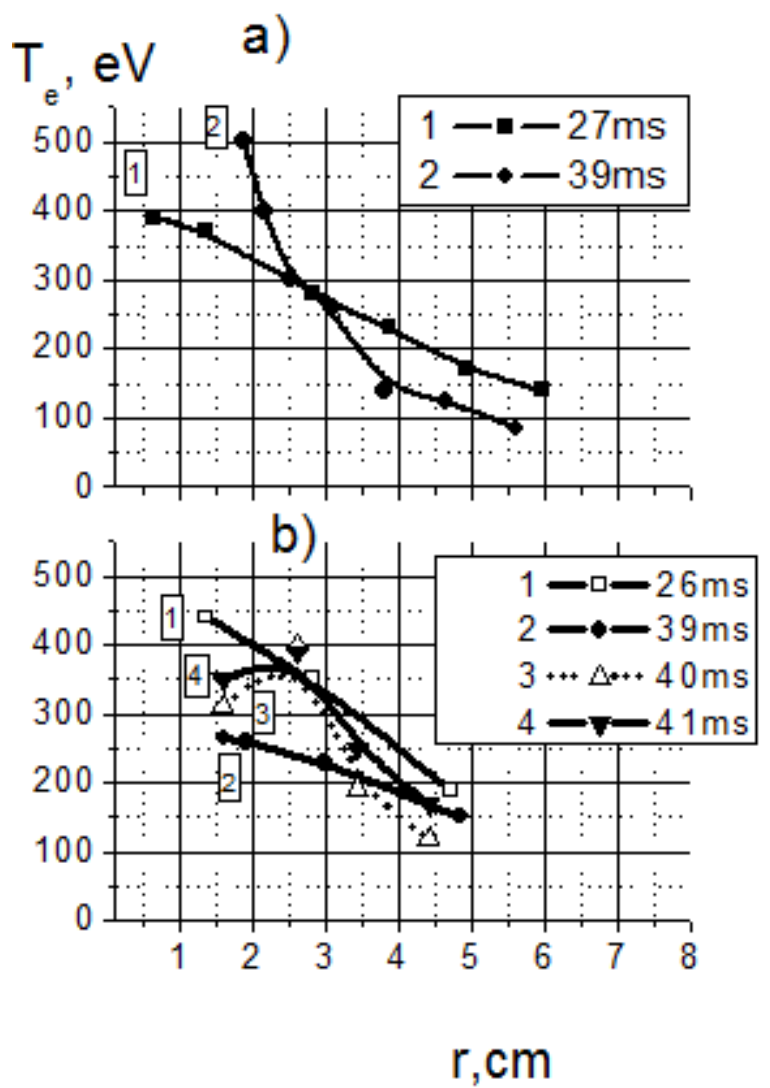

Figure 7. Electron temperature profiles obtained in two experimental regimes (with central chord plasma density variations shown in Fig. 6): (a) (regime 1) at an average electron density prior to RF pulse $\left\langle\mathrm{n}_{\mathrm{e}}\right\rangle=1.2 \times$ $10^{13} \mathrm{~cm}^{-3}$; (b) (regime 2) at an average electron density of $\left\langle\mathrm{n}_{\mathrm{e}}\right\rangle=1.4 \times 10^{13}$ $\mathrm{cm}^{-3}$

\section{About Nature of the Electromagnetic Radiation Flashes}

As is known, maser generation of the electromagnetic radiation flashes is associated with forming the region in a magnetized plasma with an inverted oscillator population having large transverse energy. Such conditions arise in the radiation belts of the Earth, Sun, planets, stars magnetosphere [15-18], as well as in laboratory plasma traps with magnetic ripples of the toroidal magnetic field in particular tokamaks with poloidal and local magnetic field ripples. In plasma traps with a mirror configuration of the magnetic field cyclotron and kinetic instabilities are created by trapped and fast electrons respectively. The buildup is accompanied by maser amplification of electromagnetic cyclotron and Alfven waves and electrostatic plasma oscillations followed by their nonlinear transformation into intense electromagnetic collective radiation (CR) [18]. 


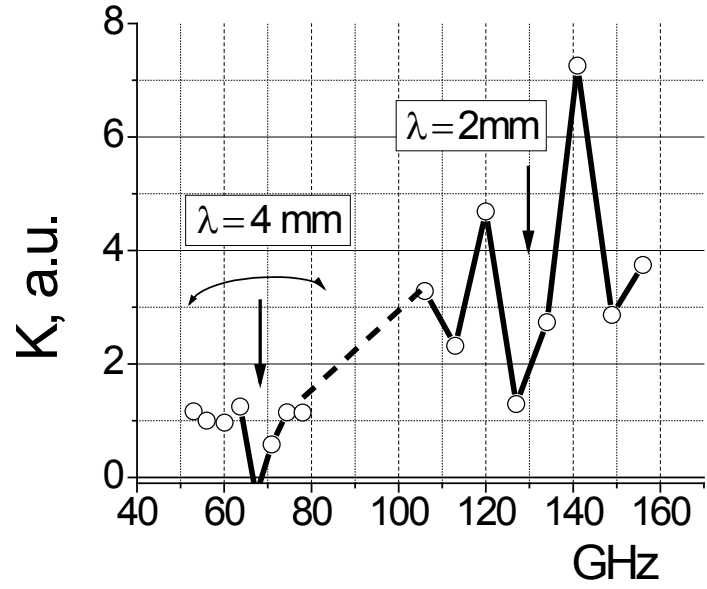

Figure 8. The relative increment of the synchrotron radiation power in LHCD. Troughs in the vicinity of the characteristic frequencies $\mathrm{f}_{\mathrm{Be}}$ and $\mathrm{f}_{\mathrm{UHR}}$, which are marked by arrows for first $(\lambda=4 \mathrm{~mm})$ and second $(\lambda=2 \mathrm{~mm})$ harmonics of SR .

Similar phenomena occur, apparently, in the laboratory plasma of magnetic bottles and tokamaks upon various instabilities created by accelerated electrons. Observations of an abnormally high level and intense flashes of microwave radiation at electron plasma and cyclotron frequencies in the Globus-M spherical tokamak OH plasma [3] and in the FT-2 tokamak $(\mathrm{OH}+\mathrm{LHCD})$ experiment $[4,6]$ have directed our efforts toward exploring the origin of maser effects that may arise in ohmically heated plasma also, in particular, upon formation of fan instability in more broader frequency diapason, [5].

\section{Data of the MR Measurements under Plasma Ohmic Heating}

Under such conditions, we investigated the MR on the FT-2 tokamak in the range of magnetic broadening of the first and second harmonics of thermal electron gyro-frequency and plasma frequency of electrons. In recording low-frequency microwave radiation, we used a direct gain receiver (DR) with detector sections for wavelengths $\lambda=3,1.5$, and $0.8 \mathrm{~cm}$ and sensitivity $P_{t h} \sim 10^{-8}$ $\mathrm{W}$ and a horn antenna located outside the discharge chamber behind a glass window pipe from the low toroidal magnetic field side. Simultaneously, we used the mentioned HRs for the 2 and $4 \mathrm{~mm}$ wavelength radiation.

This allowed us to observe the appearance and correlation of MR flashes in the frequency range of $f \sim 10 \div 40 \mathrm{GHz}$ and at higher frequencies of $69 \div 75 \mathrm{GHz}$. In these experiments, for the first time, we observed intense MR in the frequency range of $10-40 \mathrm{GHz}$ at the stage of ohmically heated plasma with moderate density of $(1.2-4) \times 10^{13} \mathrm{~cm}^{-3}$. In addition, at $<n_{e}>>2 \times 10^{13} \mathrm{~cm}^{-3}$ and frequencies $f \leq 20 \mathrm{GHz}$. The such continuous (during the discharge) radiation was accompanied by more intense giant short flashes. The results are shown in Figs. 9 to 11.
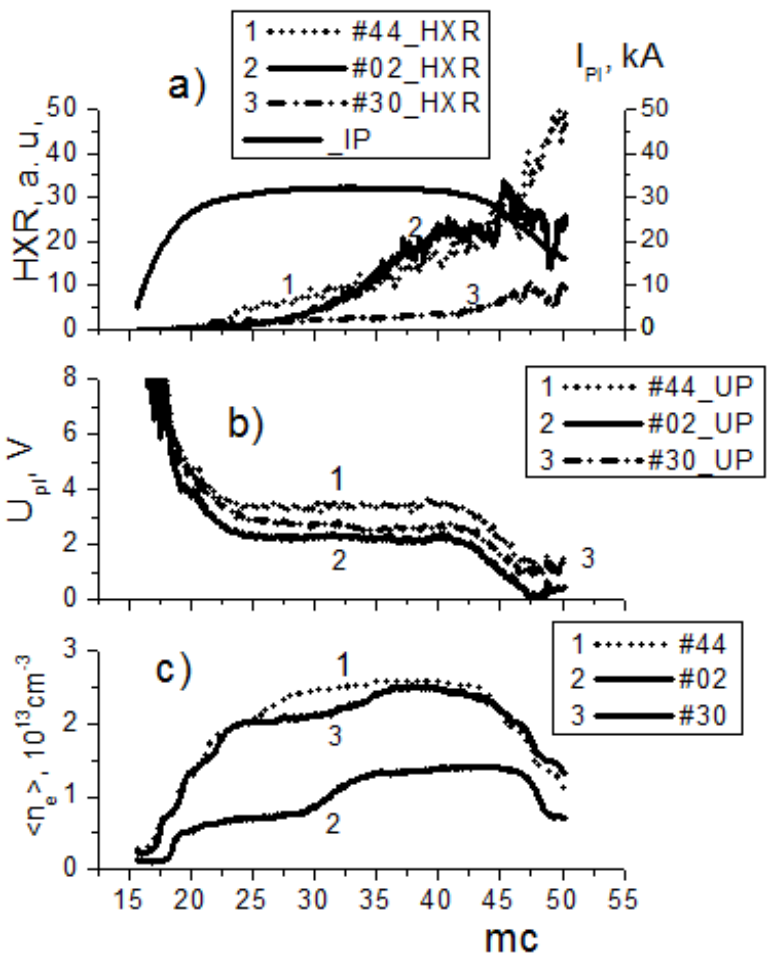

Figure 9. (a) Discharge current and intensity of the hard $\mathrm{X}$ ray radiation for discharges \#44, \#30, and \#02; (b) plasma loop voltages corresponding to these discharges; and (c) average plasma density.

$$
<n_{e}>=2.6^{*} 10^{13} \mathrm{~cm}^{-3}
$$

a)
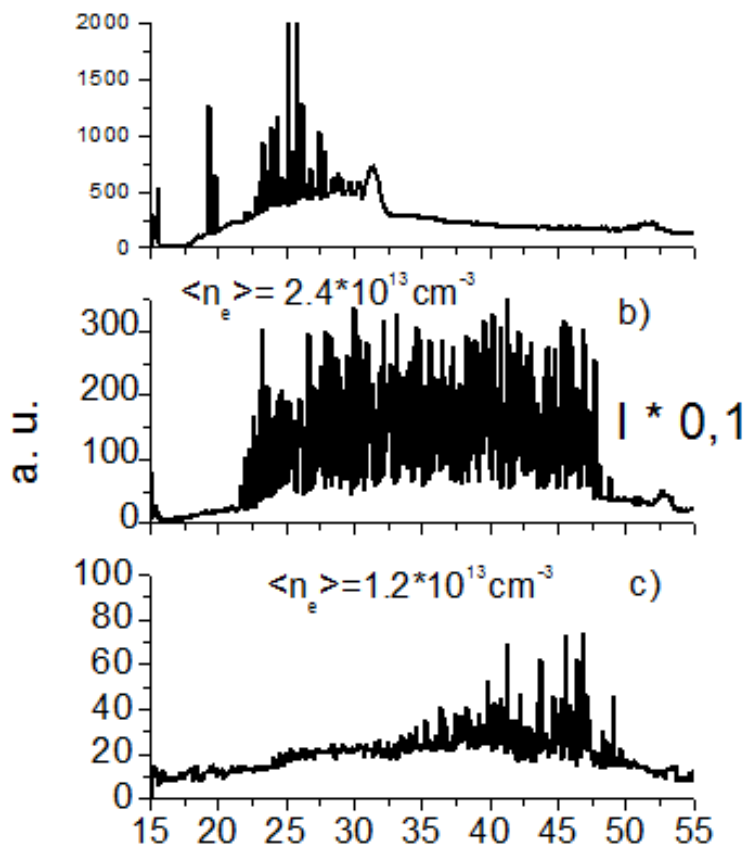

$\mathrm{ms}$

Figure 10. Temporal dependences of MR $(\lambda=3 \mathrm{~cm})$ during a discharge accompanied by short intense flashes. The data correspond to discharges (a) $\# 44$, (b) \#30, and (c) \#02. In (b), the signal is ten times reduced by multiply $\mathrm{I}^{*} 0.1$ than in (a). 


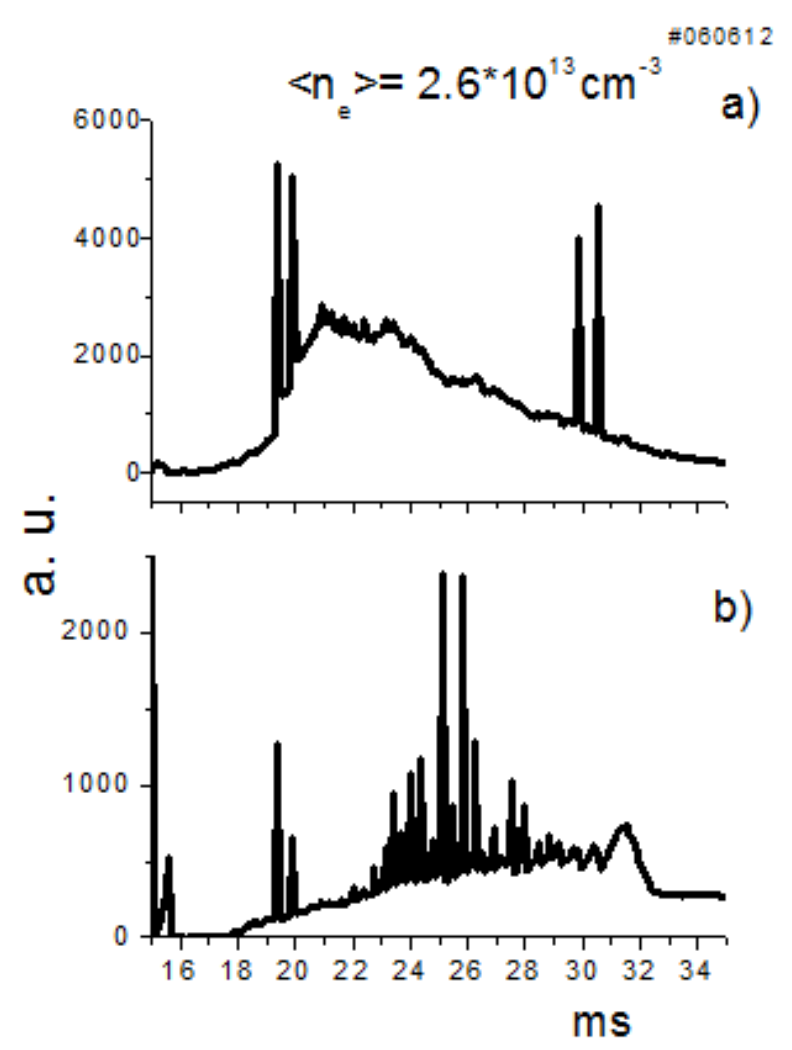

Figure 11. Temporal dependences of (a) SR $(\lambda=4 \mathrm{~mm})$ and (b) MR $(\lambda=3 \mathrm{~cm})$ registered with the heterodyne and direct gain receivers, respectively.

Figures $9 \mathrm{a}-9 \mathrm{c}$ exhibit temporal dependences of several plasma parameters for the 44th, 30th, and 2-nd discharges of the experimental series obtained on June 6, 2012. These dependences were obtained in the $\mathrm{OH}$ plasma of varying density and discharge current $\mathrm{I}_{\mathrm{pl}} \sim 30 \mathrm{kA}$ (Fig. 9a). Moreover, this figure shows the corresponding dependences of the HXR intensity. Figure $9 b$ shows dependences of loop voltage $U_{p l}$ of the plasma column for discharges of different density $<n_{e}>$, the values of which are shown in Fig. 9c. One can see that the HXR intensity increases slowly almost to the end of the discharge. It turned out that, in the low density plasma regime, the HXR intensity and magneto-hydrodynamic activity (MHD) are significantly reduced. This indirectly indicates that there is suppression of the MHD perturbations upon the fan instability forming by the current-carrying accelerated electron flow.

Figures 10a-10c show temporal dependences of the intense MR at $\lambda=3 \mathrm{~cm}$, which exists during the discharge at various plasma densities $2.6 \times 10^{13}, 2.4 \times 10^{13}$, and $1.2 \times 10^{13}$ $\mathrm{cm}^{-3}$. MR is accompanied by intense short flashes with a duration of $\sim 70 \mu \mathrm{s}$ at different stages of the discharge. It follows that a small number of large intensity flashes appear at the initial stage of the ohmic heating, when the loop voltage of the discharge chamber is rather large but plasma density is still relatively small. Considerably more such flashes appear during the quasi-stationary discharge stage in the less dense plasma $<n_{e}>=2.4 \times 10^{13} \mathrm{~cm}^{-3}$ and lower value of the vortex electric field (Fig. 10b). In the case of low plasma density $\left.<n_{e}\right\rangle=1.2 \times 10^{13} \mathrm{~cm}^{-3}$, a certain number of small flashes arise only at the end of the discharge (Fig. 10c). The same phenomena were also observed in the range of shorter wavelengths at $\lambda=1.5 \mathrm{~cm}$. The most intense flashes were found at $\left\langle n_{e}\right\rangle=2.4 \times 10^{13} \mathrm{~cm}^{-3}$. At still shorter wavelengths $(\lambda=0.8 \mathrm{~cm})$, the maximum of the MR intensity corresponds to $\left.<n_{e}\right\rangle=1.5 \times 10^{13} \mathrm{~cm}^{-3}$, whereas the flashes were observed only during the period of plasma current growth at $\left\langle n_{e}\right\rangle=2.4 \times 10^{13} \mathrm{~cm}^{-3}$ and larger values of the vortex electric field.

Figure 11 shows temporal dependences of the SR and MR intensities that were recorded using HR (at $\lambda=4 \mathrm{~mm}$ ) and DR (at $\lambda=3 \mathrm{~cm}$ ). We see that the MR that is significantly different in intensity and is also accompanied by short flashes of various intensity that occur simultaneously. This indicates the appearance of fan instability already at the discharge onset and that, in our conditions, not only does maser amplification of SR and collective radiation (CR) in the cyclotron auto- resonance mode occur, but giant flashes of the low-frequency MR appear at $\mathrm{f}<40 \mathrm{GHz}$.

Their appearance can be explained, for instance, by the self-excitation of maser amplifier at a too high magnitude of the input signal or the gain factor at a high level of the parasitic positive feedback. In the FT-2 tokamak, with local magnetic ripples of large depth and suitable parameters of the monoenergetic component of accelerated electrons, under the conditions of multiple reflection of CR from the "cut-off" surface and the corrugated wall of a discharge chamber, a maser operating in the cyclotron auto-resonance mode may be switched to the self- excitation one, when short intense quasi-cocherent MR flashes are generated. Moreover, flashes can appear simultaneously on the frequency of generation and its harmonics. Thus, an abnormally high intensity and giant flashes of the low-frequency MR discovered in our experiments can be caused by maser amplification of $\mathrm{CR}$ in the cyclotron auto-resonance mode and the self- excitation of maser amplifier, respectively.

\section{Conclusions}

MR flashes were recently registered on the FT-2 tokamak with a significantly greater depth of magnetic ripples, $\Delta B_{t} / B_{t}$ $\sim 10 \%, N_{t}=24$, in experiments on generation of the lower hybrid current drive (LHCD) in the initially ohmic heated plasma $[4,11]$. Accelerated by LHW and residual vortex electric field electrons with energy up to $4 \mathrm{MeV}$ are able to enchance fan instability and effectively interact with the local magnetic ripples in the plasma periphery.

The SR intensity increases in the frequency range corresponding to the magnetic broadening of the first and second harmonics of the gyro-frequency of thermal electrons $(55 \div 78) \mathrm{GHz}$ and $(110 \div 156) \mathrm{GHz})$ and short flashes appears. These MR bursts $(\sim 100 \mu \mathrm{s})$ appearances are caused seems by maser amplification of SR propagated in the same direction with accelerated electrons. Briefly description of mechanism of runaway electrons accumulation in the plasma 
periphery of a certain limited energy due to magnetic field ripples is presented. One proposed that the parameters of this mono-energetic beam correspond to the necessary and sufficient conditions of microwave generation on free electrons, when regime of cyclotron-auto-resonance maser, CARM, operation is realized.

The occurrence of MR flashes during a RF-pulse is followed by a fast (within 2-2.5 ms) additional heating of thermal electrons in the plasma central region $(r \leq 2 \mathrm{~cm})$ at the $\mathrm{T}_{\mathrm{e}}$ increase from 400 up to $550 \div 600 \mathrm{eV}$.

For the first time the model of the electron heating during LHCD pulse (at $\left.<n_{e}\right\rangle \approx(1 \div 1.2) \times 10^{13} \mathrm{~cm}^{-3}$ ) by Bernstein wave absorpion in the ECR region (at $\mathrm{f} \sim 60-70 \mathrm{GHz}(\lambda=4$ $\mathrm{mm})$ ) and SE one in the black plasma layers is proposed. That model can be realized in combine with improved core confinement transition [19].

For the first time, we also observed intense microwave radiation in the frequency range of $10-40 \mathrm{GHz}$ at the stage of ohmically heated plasma with moderate density of $(1.2-4) \times$ $10^{13} \mathrm{~cm}^{-3}$. In addition, at $\left\langle n_{e}\right\rangle>2 \times 10^{13} \mathrm{~cm}^{-3}$ and frequencies $f \leq 20 \mathrm{GHz}$, such a continuous (during the discharge) radiation was accompanied by more intense giant short flashes. The intense MR at $\lambda=3 \mathrm{~cm}$ exists during the plasma $\mathrm{OH}$ at various densities $2.6 \times 10^{13}, 2.4 \times 10^{13}$, and 1.2 $\times 10^{13} \mathrm{~cm}^{-3}$ which are accompanied by intense short MR flashes with a duration of $\sim 70 \mu$ s at different stages of the discharge. It turned out, that there is suppression of the MHD perturbations upon forming fan instability by the currentcarrying accelerated electrons. It turned out that, in the regime of the low density plasma, the HXR intensity and MHD activity are significantly reduced. The same phenomena of MR were also observed in the range of shorter wavelengths at $\lambda=1.5 \mathrm{~cm}$. The most intense flashes were found at $\left\langle n_{e}\right\rangle=2.4 \times 10^{13} \mathrm{~cm}^{-3}$. At still shorter wavelengths $(\lambda=0.8 \mathrm{~cm})$, the maximum of the MR intensity corresponds to $\left\langle n_{e}\right\rangle=1.5 \times 10^{13} \mathrm{~cm}^{-3}$, whereas the flashes were observed only during the period of plasma current growth at $\left\langle n_{e}\right\rangle=$ $2.4 \times 10^{13} \mathrm{~cm}^{-3}$ and larger values of the vortex electric field.

This indicates the appearance of fan instability already at the discharge onset and that, in our conditions, not only does maser amplification of $\mathrm{SR}$ and $\mathrm{CR}$ in the cyclotron auto-resonance mode occur, but giant flashes of the low-frequency MR appear at $f<40 \mathrm{GHz}$. The appearance of the giant flashes can be explained by the self-excitation of maser amplifier at a too high magnitude of the input signal or the gain factor at a high level of the parasitic positive feedback. Thus, an abnormally high intensity and giant flashes of the low frequency MR discovered in our experiments can be caused by maser amplification of CR in the cyclotron auto- resonance mode and the self- excitation of maser amplifier, respectively.

\section{Acknowledgements}

This work was supported in part by the Russian Foundation for Basic Research project nos. 13-08-00411 and
14-08-00476.

\section{REFERENCES}

[1] Kurzan, B. and Steuer, K.H., Runaway electrons in a tokamak: A free electron maser, Phys. Rev., E 55, 4608, (1997).

[2] Kurzan, B., Steuer, K.H., and Fussmann, G., Dynamics of Runaway Electrons in the Magnetic Field of a Tokamak. Phys. Rev. Lett., 75, (25), 4626, (1995).

[3] Anan'ev, A.S, Gusev, V.K., Krikunov, S.V., et al., Nonthermal microwave radiation under plasma ohmic heating in the spherical Globus-M tokamak, Proc.12th Joint Workshop on ECE \& ECRH (Aix-en-Provence, France), 13-16 May 2002, p.191.

[4] Lashkul, S.I., Rozhdestvenskii, V.V., Altukhov, A.B., et al., Characteristic features of accelerated electrons on Lower Hybrid Current Drive experiments in the FT-2 tokamak. Tech. Phys. Lett., 38, (12), 1073, (2012).

[5] Rozhdestvensky, V.V., Krikunov, S.V., Lashkul, S.I., et al. Features of the microwave radiation under the fan instability development and accelerated electron interaction with magnetic ripples in the FT- 2 tokamak ohmically heated plasma. Tech. Phys. Lett. 40, (5), pp. 43-50, (2014).

[6] Budnikov, V.N., Dyachenko, V.V., Esipov, L.A., et al. Cyclotron and collective microwave emission under ohmical heating and low-hybrid pumping of the FT-2 tokamak plasma., Proc. 10th Joint Workshop on ECE \& ECRH (Ameland, the Netherlands), 6-11 April 1997, p. 427.

[7] Laurent, L., and Rax, J.M., Stochastic Instability of Runaway Electrons in Tokamaks, Europhys. Lett. 11, 219, (1990).

[8] Russo, A. J., Effect of ripple on runaway electrons in tokamaks, Nucl. Fus. 31, 117 (1991).

[9] Martin-Solis, J.R., Esposito, B., Sanchez, R., and Alvarez, J.D., Energy limits on runaway electrons in tokamak plasmas, Phys. Plasmas 6 (1), 238, (1999).

[10] Parail, V.V. and Pogutse, O.P., Accelerated electrons in tokamaks, in Reviews of Plasma Physics, Ed. by Leontovich, M.A. and. Kadomtsev, B.B. (Energoizdat, Moscow, 1982).

[11] Lashkul, S.I., Altukhov, A.B., Gurchenko, A.D., et al., Analysis of the efficiency of lower hybrid current drive in the FT-2 tokamak Plasma Phys. Rep., 36, (9), 751, (2010).

[12] Shevelev, A.E., Khilkevitch, E.M., Kiptily V.G. et al., Reconstruction of distribution functions of fast ions and runaway electrons in fusion plasmas using gamma-ray spectrometry with applications to ITER. Nucl. Fusion 53 (2013) 123004 (12pp).

[13] Chen, Z.Y.,. Wan, B.N, Lin, S.Y., et al. Interaction of Runaway Electrons with Magnetic Field Ripple in the HT-7 Tokamak. Proceedings of the 21st IAEA Fusion Energy Conf.(October 16-21, 2006, Chengdu, China), EX/P3-4.

[14] V. E. Golant and V. Fedorov, Nethods of High_Frequency Plasma Heating in Toriodal Thermonuclear Fusion Setups (Moscow, 1968), Ch. 3. 
[15] Bespalov, P. A. and Trakhtengerts, V.Yu. Alfven Masers (Gorky, 1986).

[16] Trakhtengerts, V.Yu.and Demekhov, A.G. Cosmic cyclotron masers. Priroda 4, (2002).

[17] Fleishman, G.D. and Mel'nikov, V.F., Millisecond solar radio spikes, Phys. Usp. 41, (12), 1157, (1998).

[18] Zhelezniakov, V.V. Radiation in Astrophysical Plasmas. Kluwer, 1996.

[19] Lashkul, S.I, Altukhov, A.B, Gurchenko, A.D. et al., Impact of isotopic effect on density limit and LHCD efficiency in the FT-2 experiments, Nucl. Fusion, 55, (2015), 073019 